\title{
ACSNANO
}

\section{Standing Enokitake-like Nanowire Films for Highly Stretchable Elastronics}

3 Yan Wang, ${ }^{\dagger, \text { Il }}$ Shu Gong, ${ }^{\dagger, \text {,I }}$ Stephen J. Wang, ${ }^{\|, \perp}$ Xinyi Yang,, Yunzhi Ling, ${ }^{\dagger}$ Lim Wei Yap, ${ }^{\dagger \oplus}$

${ }_{4}$ Dashen Dong, ${ }^{\dagger}$ George P. Simon, ${ }^{\S}$ and Wenlong Cheng ${ }^{*, t, \neq}$

$5{ }^{\dagger}$ Department of Chemical Engineering, ${ }^{\S}$ Department of Materials Science and Engineering, and ${ }^{\|}$International Tangible Interaction

6 Design Lab, Monash University, Clayton, Victoria 3800, Australia

${ }_{7}{ }^{\ddagger}$ The Melbourne Centre for Nanofabrication, Clayton, Victoria 3800, Australia

${ }_{8}^{\perp}$ Department of Innovation Design Engineering, School of Design, Royal College of Art, London SW7 2EU, United Kingdom

9 S Supporting Information

10 ABSTRACT: Stretchable electronics may enable electronic 11 components to be part of our organs-ideal for future 12 wearable/implantable biodiagnostic systems. One of key 13 challenges is failure of the soft/rigid material interface due 14 to mismatching Young's moduli, which limits stretchability 15 and durability of current systems. Here, we show that 16 standing enokitake-like gold-nanowire-based films chemi17 cally bonded to an elastomer can be stretched up to $900 \%$ 18 and are highly durable, with $>\mathbf{9 3} \%$ conductivity recovery
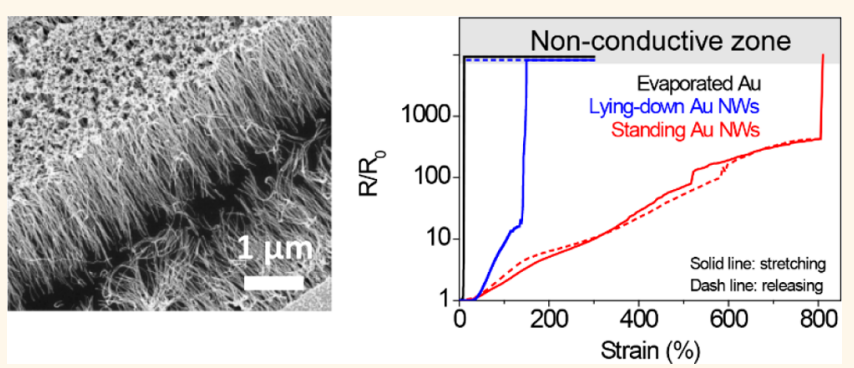
even after 2000 stretching/releasing cycles to $800 \%$ strain.

Both experimental and modeling reveal that this superior elastic property originates from standing enokitake-like nanowire film structures. The closely packed nanoparticle layer sticks to the top of the nanowires, which easily cracks under strain, whereas the bottom part of the nanowires is compliant with substrate deformation. This leads to tiny $\mathrm{V}$ shaped cracks with a maintained electron transport pathway rather than large U-shaped cracks that are frequently observed for conventional metal films. We further show that our standing nanowire films can serve as current collectors in supercapacitors and second skin-like smart masks for facial expression detection.

KEYWORDS: standing nanowire film, unconventional crack, elastronics, electronic skins, strain sensors

27
28 lectronics are transitioning from the current rigid version to a next-generation flexible design, which will ultimately evolve into stretchable electronics (i.e., 30 elastronics). In an elastronic system, its components can be 31 seamlessly integrated with skin/muscles to become parts of our 32 organs, thereby enabling genuine biodiagnostics in real time 33 and in situ. It is well-known that elastronics requires a seamless 34 combination of stretchability and electrical conductivity, which 35 can be achieved extrinsically or intrinsically. ${ }^{1-3}$ The former is 36 achieved by designing structures that stretch, ${ }^{4-10}$ whereas the 37 latter is realized by producing materials that are deform38 able. $^{11-22}$

39 An ideal elastronic system may be made from intrinsically 40 elastic components, including conductors, resistors, diodes, 41 transistors, and sensors, so that they can integrate with 42 modulus-matching skin/muscle, ${ }^{12-19,23,24}$ ideal for wearable/ 43 implantable diagnostics with true capability of health 44 monitoring anytime and anywhere. A viable strategy is to 45 deposit active nanomaterials onto or embed them into 46 elastomers. ${ }^{12,14-17,25-33}$ Among them, one-dimensional nano47 materials are particularly promising as they can be used to 48 construct percolation networks onto or into elastomeric matrices. ${ }^{12,14,22,25-32,34}$ Two-dimensional (2D) percolation 49 nanowire-based thin films have demonstrated a wide range of 50 applications in wearable electronic skin (e-skin) sensors, ${ }^{33}$ soft 51 energy devices, ${ }^{36,37}$ organic light-emitting diodes, ${ }^{38}$ memory 52 devices, ${ }^{39}$ PM 2.5 filters, ${ }^{40}$ soft robotics, ${ }^{26}$ and transparent 53 electronics. ${ }^{41-46}$ Despite this encouraging progress, delamina- 54 tion and/or cracks at the soft/rigid materials' interface often 55 occur under large or repeated strains due to mismatching 56 Young's moduli between active rigid materials and soft 57 elastomeric matrixes. This limits the stretchability and long- 58 term durability of current systems, preventing them from being 59 used in real-world applications. ${ }^{47}$

In this work, we show that standing enokitake-like gold 61 nanowire films chemically bonded to elastomeric materials can 62 exhibit stretchability (up to 900\%) much higher than that of 63 conventional vacuum-evaporated bulk metal or percolating 64 nanowire films, without any additional extrinsic buckling 65 design. This was achieved because of standing enokitake-like 66

Received: July 3, 2018

Accepted: September 4, 2018 

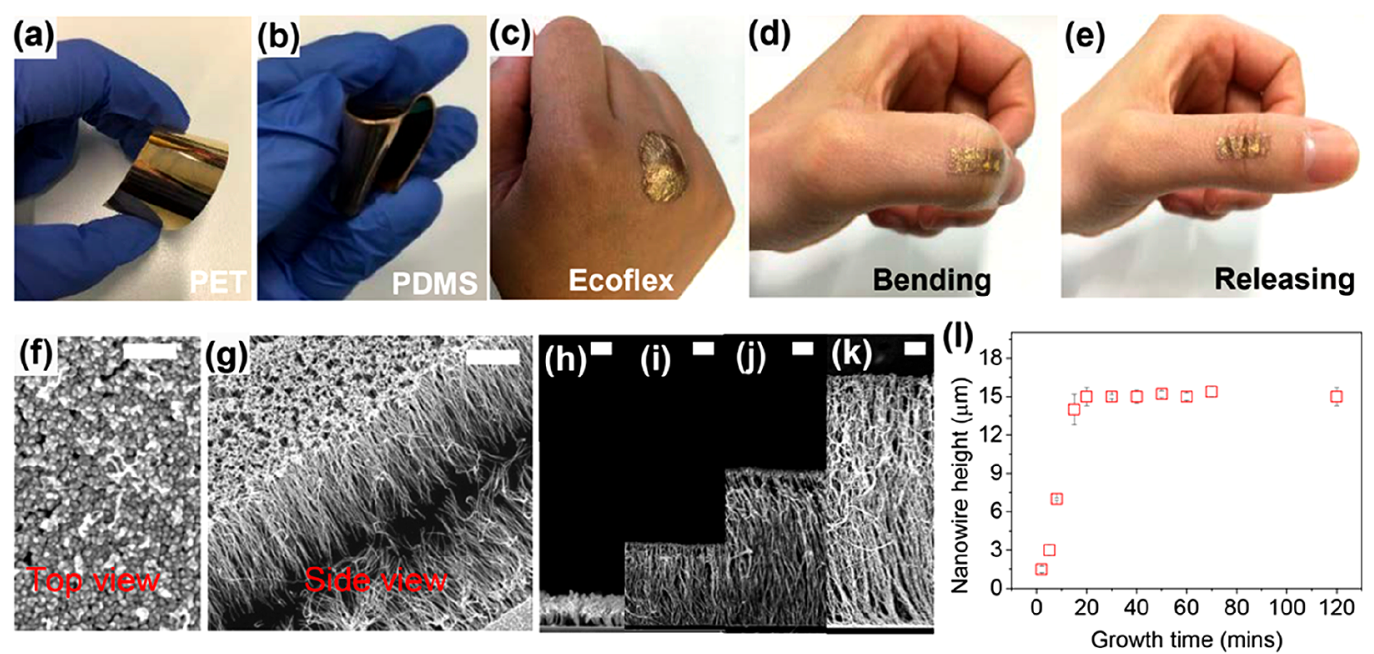

Figure 1. Characteristics of standing enokitake-like nanowire-based gold films. $(a-c)$ Optical appearances of standing enokitake-like nanowire-based films grown on flat elastomers: (a) PET, (b) PDMS, (c) Ecoflex. (d,e) Photographs of the thin standing enokitake-like nanowire-based gold films with skin-textured Ecoflex substrate on a human thumb knuckle while bending and releasing, respectively. (f,g) Typical top-view and side-view SEM image of standing enokitake-like nanowire-based gold films. Scale bar: $200 \mathrm{~nm}$. (h-k) SEM images of standing nanowire films with different thicknesses: (h) $\sim 1.5 \mu \mathrm{m},(\mathrm{i}) \sim 3.5 \mu \mathrm{m},(\mathrm{j}) \sim 7 \mu \mathrm{m}$, and (k) $\sim 14 \mu \mathrm{m}$. Scale bar: $1 \mu \mathrm{m}$. (1) Change of nanowire height as a function of growth time.

67 nanowire structures and their strong adhesion with elastomers, 68 leading to distinct stretching behaviors. Unlike conventional 69 metal films (by vacuum evaporation/sputtering or previous 70 nanomaterials films) which typically exhibit large "cliff-like" 71 "U-shaped" cracks that cannot recover upon releasing the 72 strain, our standing enokitake-like nanowire gold films instead 73 show tiny "V-shaped" cracks that are able to recover the 74 conductivity when strain is removed. The formation of V75 shaped cracks is due to hierarchical structures of the nanowire 76 film, in which the top nanoparticle layer is mechanically more 77 rigid than the underlying nanowire layer. This leads to initial 78 cracking that starts from the top particle layer under low level 79 of strains (typically below $300 \%$ ), followed by conventional 80 large U-shaped cracks of the entire film under large strains 81 (typically between 300 and $800 \%$ ). In both cases, conductivity 82 pathways could be maintained. This unconventional property 83 enables our enokitake-like nanowire film to be used as highly 84 durable conductors which could retain the $>93 \%$ conductance 85 even after 2000 stretching/releasing cycles to $800 \%$ strain. We 86 demonstrate specifically here that they can be applied to 87 fabricate intrinsically stretchable supercapacitors and can be 88 used as "second-skin" facial expression recognition mask 89 sensors.

\section{RESULTS AND DISCUSSION}

91 By extending the method of seed-mediated electroless plating 92 on rigid surfaces, ${ }^{48}$ standing enokitake-like nanowire-based 93 gold films could grow on a number of polymer substrates 94 including polyethylene terephthalate (PET), polydimethylsi95 loxane (PDMS), and Ecoflex (highly stretchy silicone rubber). 96 Macroscopically, the standing nanowire films were uniform 97 with a shiny gold reflective surface if the underlying 98 elastomeric substrates were flat (Figure $1 \mathrm{a}-\mathrm{c}$ ). The fabrication 99 process is illustrated in Figure S1. In brief, an elastomeric 100 substrate is first treated using $\mathrm{O}_{2}$ plasma to render its surface 101 hydrophilic, which is then followed by silanization with (3102 aminopropyl)trimethoxysilane (APTMS). Next, negatively 103 charged seed particles could be immobilized onto this amine104 functionalized surfaces via electrostatic attraction. Further immersion of the seed-particle-modified elastomer into a 105 growth solution containing gold precursors, surfactants, and 106 reducing agents could lead to the formation of densely packed 107 standing nanowire arrays. The gold films grown on thin Ecoflex 108 sheets $(\sim 20 \mu \mathrm{m}$ thickness) could naturally attach to human 109 skin wrinkles before and after stretching (Movie S1). The 110 growth process was found to be scalable and able to 111 conformably coat a range of other polymer substrates from 112 macroscopic to microscopic (Figure S2a-f) and even to 113 textured skin replicas (Figure 1d,e and Figure S2g). Superior 114 skin conformal attachment in conjunction with chemical 115 inertness and biocompatibility of gold indicates the great 116 potential of our nanowire film as second skin patches for 117 various biomedical applications.

118

Further top-view and side-view characterizations by scanning 119 electron microscopy (SEM) revealed enokitake-like nanowire 120 film structures (Figure 1f,g), in which the top layer ("head" 121 side) consists of closely packed gold nanoparticles with a 122 diameter of $9.3 \pm 2.1 \mathrm{~nm}$. The bottom layer ("tail" side) is 123 composed of nanowires standing normal to the elastomer 124 substrates, with a typical nanowire diameter of $7.8 \pm 1.7 \mathrm{~nm}$. In 125 addition, the number density of nanowires can reach as high as 126 $\sim 1.09 \times 10^{4} \mu \mathrm{m}^{-2}$, which is much higher than that of 127 previously reported $2 \mathrm{D}$ nanowire percolation network 128 systems. $^{35-39}$ The estimated porosity of the head side is 65- 129 $72 \%$, whereas the tail side is $50-55 \%$. Longer growth times 130 lead to longer nanowires but reach the plateau in about $20 \mathrm{~min} 131$ (SEM images in Figure $1 \mathrm{~h}-\mathrm{k}$ ). We obtained nanowires that 132 were much longer than those in the literature ${ }^{48}$ by using 133 concentrated growth solution to achieve tunable lengths up to 134 $\sim 15 \mu \mathrm{m}$ (Figure 11). In addition, the diameter of both 135 nanoparticle and nanowire did not change much as the 136 nanowire became longer (Figure S3). It is even possible to 137 grow staircase-like nanowire films by mask-assisted step growth 138 (Figure S4). Overall, the structural features including accurate 139 height control, standing enokitake-like configuration, and 140 control over surface topological structures indicate that our 141 system is different from a dominant nanowire percolation 142 

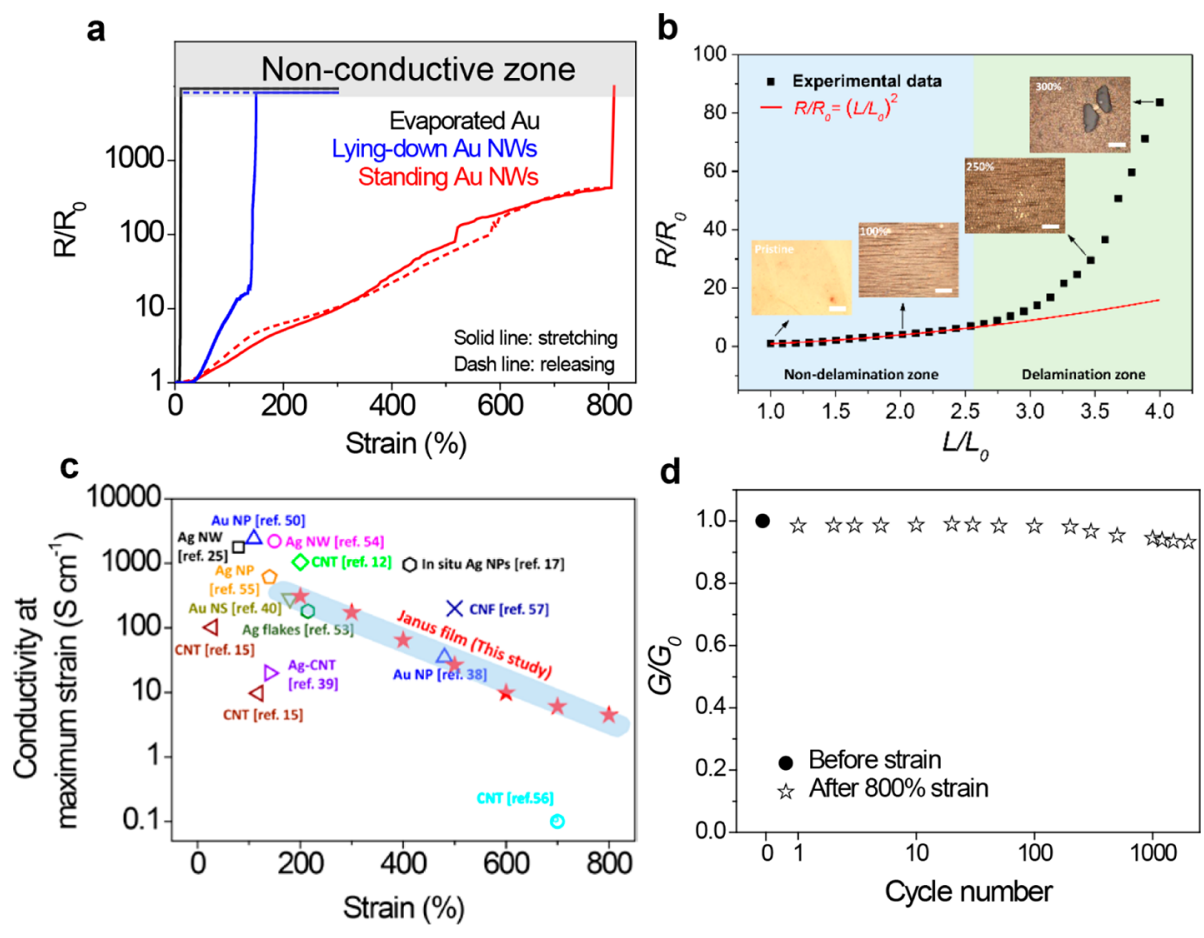

Figure 2. Superior intrinsic stretchability of standing enokitake-like nanowire-based gold films. (a) Comparison of stretchability among evaporated Au films, lying-down gold nanowire film and standing enokitake-like nanowire-based gold film. (b) Plot of normalized resistance $\left(R / R_{0}\right)$ versus normalized length $\left(L / L_{0}\right)$. Scattered black squares denote experimental data; the red curve is the theoretical prediction based on the equation $R / R_{0}=\left(L / L_{0}\right)^{2}$. Inset: Representative optical images of standing nanowire film under different strains of 0 , 100, 250, and $300 \%$. Scale bar: $20 \mu \mathrm{m}$. (c) Comparison of this work to recent work in elastic conductors. Data points are extracted from the following papers: blue open triangle, Au nanoparticles (Au NPs); ${ }^{50}$ pink open circle, Ag nanowires (Ag NWs); ${ }^{54}$ black open square, Ag NWs; ${ }^{25}$ lime open diamond, carbon nanotube (CNT); ${ }^{12}$ black open pantagon, in situ Ag NPs; ${ }^{17}$ orange open pentagon, Ag nanoparticles (Ag NPs); ${ }^{55}$ pistachio open inverted triangle, Au nanosheets (Au NSs); ${ }^{40}$ Royal cross, carbon nanofibers (CNFs); ${ }^{57}$ green open pantagon, Ag flakes; ${ }^{41}$ sienna left open triangle, CNT; ${ }^{15}$ purple open right triangle, Ag carbon nanotubes (Ag CNT); ${ }^{40}$ cyan open circle, CNT; ${ }^{56}$ red filled star, this study. (d) Conductance change of standing enokitake-like nanowire-based film during 2000 stretching/releasing cycles up to $800 \%$ strain.

143 network $^{26,35-39,46}$ and may be viewed as a three-dimensional 144 percolation system.

145 We systematically investigate stretchability of the standing 146 nanowire-based film. When directly grown on Ecoflex 147 substrates with the nanowire chemically bound to surfaces, 148 the films exhibit exceptionally high stretchability up to $800 \%$ of 149 strain (Figure 2a, red solid line). With additional Ecoflex 150 encapsulation, the conductivity was observed to survive even at 151 the $900 \%$ strain, which is almost the physical limit of the 152 Ecoflex elastomer (Figure S5). The improved stretchability 153 with Ecoflex encapsulation may be due to the enhanced 154 bonding at the top side, leading to more uniform crack 155 propagation of the nanoparticle, preventing catastrophic 156 failure. This observation is in agreement with sandwiched 157 silver-nanowire-percolated structure reported previously. ${ }^{49}$ 158 Remarkably, the original conductivity could be recovered 159 upon stress release (Figure 2a, red dashed line). In control 160 experiments, we found that the evaporated gold can only 161 survive $\sim 10 \%$ strain before conductivity is lost, and the 162 percolation lying-down nanowire film is only able to tolerate a $163 \sim 150 \%$ strain (blue solid line in Figure $2 \mathrm{a}$ ). Both bulk metal 164 and percolation nanowire films show no conductivity recovery 165 upon stress release (black and blue dashed lines in Figure 2a). 166 We further plot normalized resistance $\left(R / R_{0}\right)$ versus 167 normalized length square $\left(L / L_{0}\right)^{2}$ for experimental data 168 collection and theoretical prediction (Figure $2 \mathrm{~b}$ ), where $R_{0}$ 169 and $L_{0}$ are the resistance and length, respectively, of samples at $1700 \%$ strain. The deviation starts at a strain of $\sim 150 \%$, above which cracks form and propagate, which is further validated 171 from optical imaging (inset of Figure $2 \mathrm{~b}$ ). This threshold value 172 is 3-fold that for copper-bonded Kapton film. ${ }^{47}$ Note that 173 $800 \%$ stretchability for an enokitake-like standing nanowire 174 film outperforms the state-of-the-art inorganic stretchable 175 conducting film ${ }^{12,15,17,25,50-57}$ (Figure 2c). Remarkably, the 176 film conductance $G$ retained $>93 \%$ of the initial conductance 177 $\left(G_{0}\right)$ after stretching/releasing to $800 \%$ strain for 2000 cycles 178 (Figure 2d). This has not yet been achieved, to the best of our 179 knowledge, by previously reported stretchable conductors 180 without using prestrain or buckling designs.

We further established that strong adhesion between the 182 nanowire and Ecoflex substrate and "accordion-fan-like" V- 183 shaped cracking processes is responsible for the exceptional 184 high stretchability observed. The adhesion test (Movie S2) 185 clearly shows that our standing enokitake-like nanowire film 186 could survive in the normal Scotch tape test multiple times 187 without significant resistance change. The strong adhesion may 188 be due to the use of APTMS that serves a bifunctional 189 molecular glue. Its amine side strongly interacts with gold 190 nanowires, and its silane sides covalently bond to Ecoflex 191 surfaces. The introduction of an organic intermediate layer has 192 been demonstrated as an effective strategy to improve the 193 adhesion between the metallic layer and polymeric substrates, 194 thus enhancing the overall performance of the stretchable 195 conductive film. $^{58-61}$ Unlike the continuous bulk metal film, 196 our nanophased enokitake-like structures offer better stretch- 197 ability (Table S1). 


\section{$\varepsilon=0 \%$}

(a)

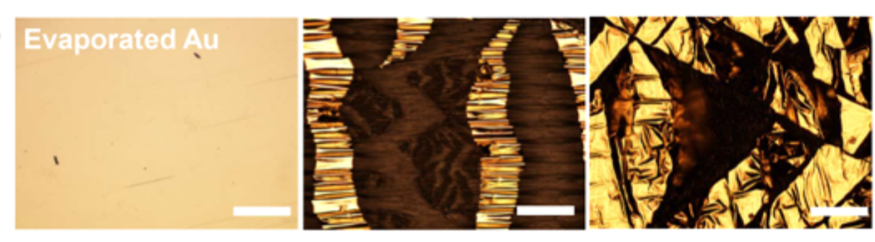

(c)

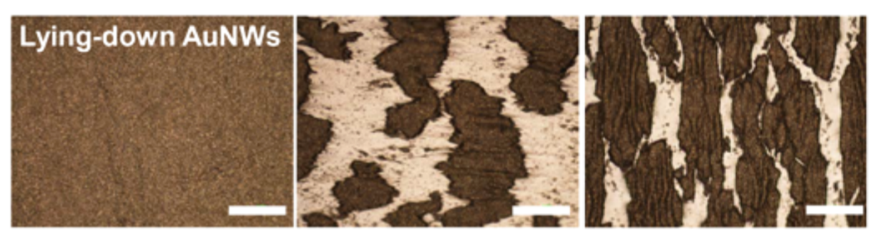

(e)

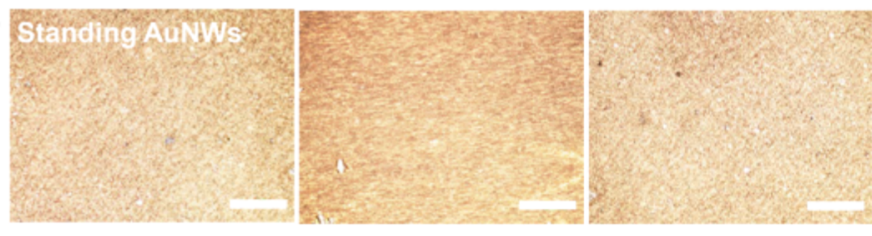

AFM at $\varepsilon=300 \%$

(b)
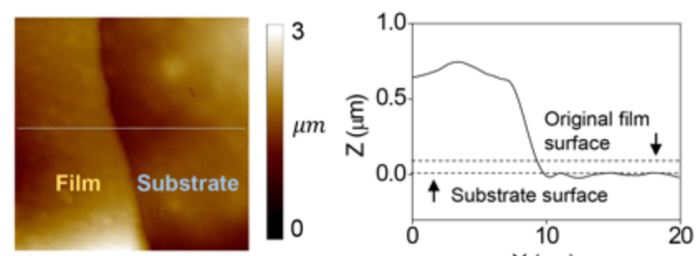

(d)

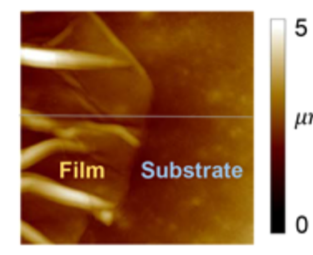

(f)

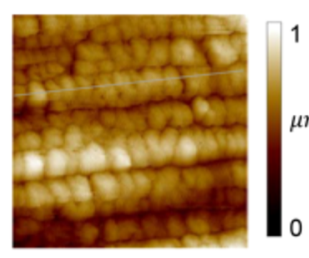

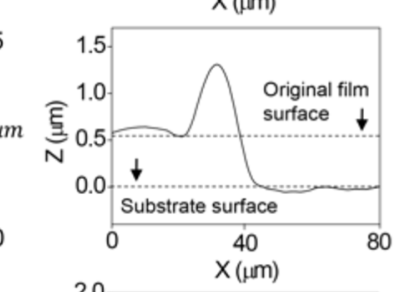

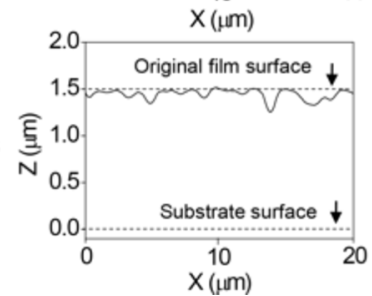

Figure 3. Optical microscopic and AFM characteristics of three different gold films (evaporated gold film, lying-down nanowire gold film, and standing enokitake-like nanowire-based gold film). Microscopic behavior of (a) evaporated Au film, (c) lying-down nanowire gold film, and (e) standing enokitake-like nanowire-based gold film by optical microscope imaging at various strain (from 0 , 300, and back to $0 \%$ ), respectively. AFM images and height plots of (b) evaporated gold film, (d) lying-down nanowire gold film, and (f) standing enokitake-like nanowire-based gold film under $300 \%$ strain. Nanowire height for standing enokitake-like nanowire-based gold film is $1.5 \mu \mathrm{m}$. Scale bar: 200 $\mu \mathrm{m}$. All optical images have the same resolution.

199 Unlike conventional bulk gold or percolation nanowire films, 200 our standing nanowire films have hierarchical structures with 201 close-packed nanoparticle arrays on the top and aligned 202 nanowires chemically bound to elastomeric substrates. This 203 leads to a distinct stretching mechanism (Figure S6). For 204 further investigation, we carried out detailed multiscale 205 morphological studies in order to understand the exceptional 206 stretchability observed. We scrutinized morphological features 207 in different locations of rectangle standing nanowire metallic 208 nanopatches under various strains by optical microscopy 209 (Figure S7). This offers a panoramic overview of our standing 210 nanowire film stretching process at millimeter and micrometer 211 length scales. Evident cracks will not be seen until about $300 \%$ 212 strain is applied. At the nanoscale, atomic force microscopy 213 (AFM) and cross-sectional SEM characterization under a 214 stretched state clearly show the presence of V-shaped cracks 215 (Figures S8 and S9). The cracking depths measured for the 216 two particular standing nanowire films under different strains 217 were significantly lower than the film thickness. Assuming that 218 the nanowire deforms elastically without breaking up and with 219 its ends firmly attached to elastomeric substrates, we can 220 visualize a $\mathrm{V}$-shaped cracking process by finite element analysis 221 (Movie S3). However, both bulk gold films and percolation 222 nanowire films exhibit only typical U-shaped cracks (Figure $2233 \mathrm{a}-\mathrm{d}$; also see Figure S10 for the schematic illustration of V224 shaped crack and U-shaped crack). Both can tolerate a level of 225 strain much less than that for the standing nanowire films. The 226 concurrent film delamination prevents recovery of original 227 structures, hence, leading to poor conductivity recovery 228 (Figure S6a,b). Note that the stretching mechanism of our 229 nanowire film is fundamentally different from previous aligned 230 carbon nanotube arrays where building blocks were not standing normal to the substrate but were lying down flush on 231 the substrate. ${ }^{30}$

The above multiscale structural characterizations and finite 233 elemental analysis reveal the following mechanistic insights. 234 Cracks initiate from the head side, which serve as unzipping 235 points for strongly bundling nanowire arrays, yet the 236 interacting nanowire tail ends deform conformably to the 237 substrate without cracking (Figure 3e,f and Figure S6c). At the 238 point when substrate elongation commences, the mechanically 239 rigid top gold nanoparticle layer (head side) cracks, which 240 triggers the formation of $\mathrm{V}$-shaped cracks as the strain level is 241 increased by unzipping them from the top side. This typically 242 occurs when the strain level is less than $\sim 150 \%$ strain, where 243 no delamination occurs between substrates and our gold film at 244 this stage. Obvious wrinkles are observed in the middle part of 245 the standing nanowire film because of the Poisson ratio of 246 Ecoflex substrate (Figure S7, middle left). As the strain 247 increases further to a certain threshold, large U-shaped cracks 248 form as a result of the standing nanowire film sliding/ 249 delaminating from the supporting elastomeric substrates. The 250 U-shaped cracks propagate as the strain level is further 251 increased; however, percolation conductive pathways are still 252 maintained until reaching a catastrophic failing point. The V- 253 shaped and U-shaped cracks coexist at the high strain levels 254 typically from 300 to $800 \%$. The self-repairable cracks were 255 also demonstrated from more detailed SEM characterization. 256 By inspecting the same spot in a particular sample, negligible 257 morphological changes were observed before and after 60000258 cycles of stretching/releasing to $185 \%$ strain (Figure S11). Its 259 excellent stretchability was maintained even after 40 weeks of 260 storage in ambient conditions without encapsulation (Figure 261 S12). 

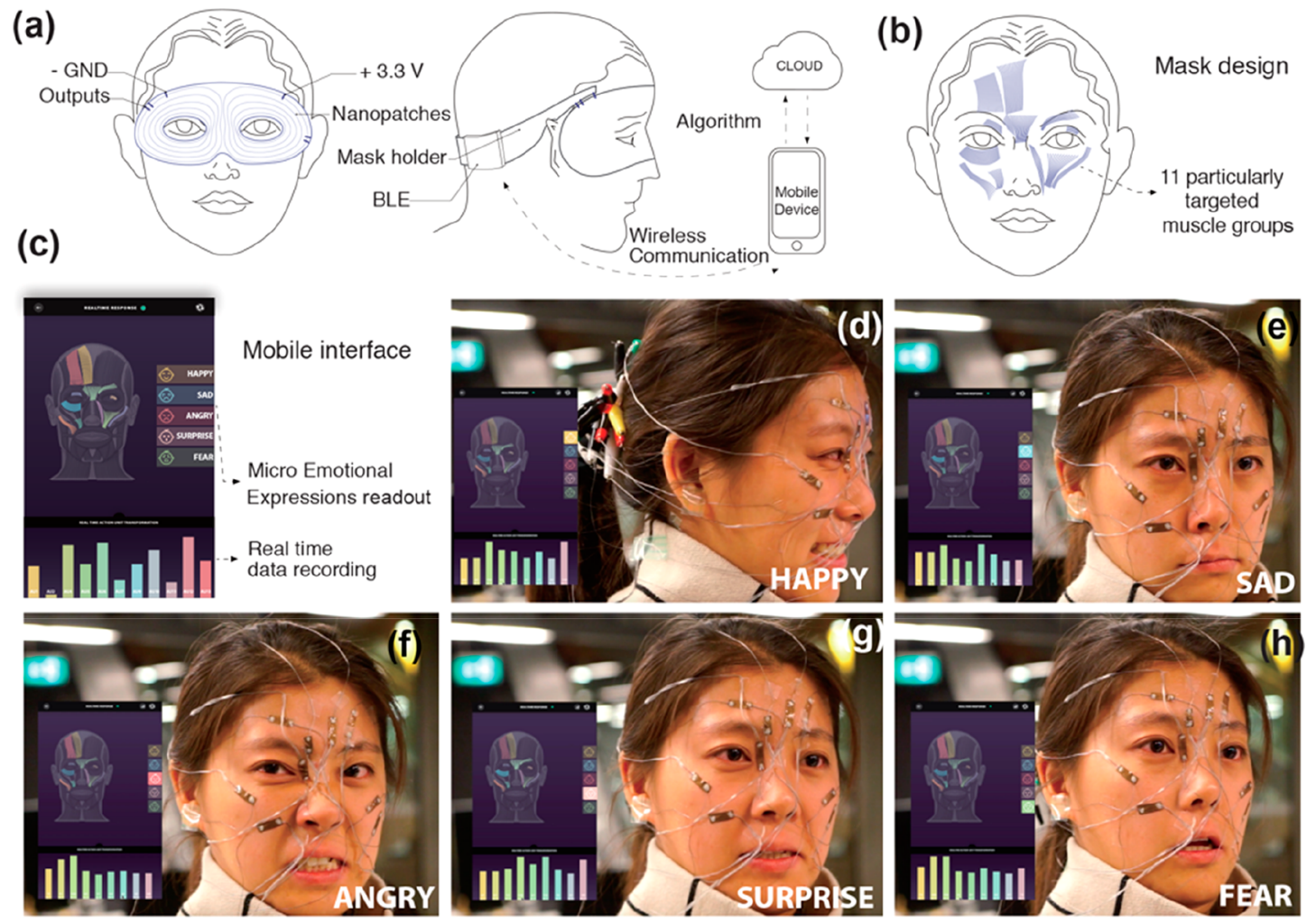

Figure 4. Real-time facial expressions monitoring. (a) Schematic illustration of the detection system setup. (b) Schematic of standing enokitake-like nanowire-based gold film smart mask design according to nine facial muscle group movements caused by various emotions. (c) Mobile device interface for result reading. (d-h) Real-time monitoring of five different facial expressions of happy, sad, angry, surprise, and fear.

263 We also found that the stretchability of the standing 264 nanowire film showed a decreasing trend, whereas nanowire 265 length increased (Figure S13a). As the nanowire length 266 increased to $14 \mu \mathrm{m}$, the film lost conductivity at $80 \%$ strain, 267 which is 10 times lower than that of the $1.5 \mu \mathrm{m}$ film. As 268 expected, the overall nanowire/Ecoflex sheet became stiffer as 269 the nanowire length increased (Figure S13b). This could be 270 due to strong wire-to-wire interactions among longer nano271 wires, rendering nanowire films more rigid, approaching bulk 272 gold mechanical properties.

273 The facile growth of a standing nanowire film in conjunction 274 with their outstanding performances indicates their suitability 275 for soft electronics applications. As the first proof of concept, 276 we demonstrate their use in soft, stretchable supercapacitors 277 using our gold film with short nanowires. In a typical 278 symmetrical layout, we were able to achieve excellent 279 capacitive behavior (Figure S14), which also shows negligible 280 changes over a wide range of applied tensile strain from 0 to $281250 \%$. The slight capacitance increase from 0 to $100 \%$ strain 282 may be due to increased surface area of the nanowire 283 unzipping process under strain. Further stretching beyond 284 the $100 \%$ strain caused a very small decrease in the 285 capacitance, retaining $84 \%$ of the original capacitance at a 286 strain up to $250 \%$ (Figure S15a,b). This slight degradation of 287 capacitance is possibly due to the conductivity decreases of 288 standing nanowire film electrodes and/or deformation of the 289 electrolyte layers over stretching. Nevertheless, specific 290 capacitance could be maintained by $99 \%$ after 200 stretch/ 291 release cycles at the strain of $200 \%$, suitable for wearable on292 body energy storage devices (Figure S15c,d).
The excellent skin conformability of our standing nanowire 293 film enabled its use as e-skin smart nanopatches for detecting 294 childhood autism disorder. Note that the smart nanopatches 295 were fabricated by a strain-sensitive film from longer standing 296 nanowires. Instead of an optical approach used by the NODA 297 diagnostic tool available on Apple store, we used nine e-skin 298 nanopatches to monitor particular pieces of muscle/skin 299 stretching related to facial expression (Figure 4). Based on $300 \mathrm{f} 4$ the information from the Facial Action Coding System 301 (FACS) library from Ekman's group, ${ }^{62}$ we could relate 302 electrical signals to the five different emotional expressions 303 (happy, sad, angry, surprise, and fear) in a wireless manner 304 (Movie S4). Different facial expressions can be read from a 305 mobile screen in real time.

\section{CONCLUSIONS}

307

In summary, we report the exceptional high stretchability and 308 durability of standing enokitake-like nanowire-based gold films, 309 which are unexpected in the context of current dominant 310 nanowire percolation network-based stretchable conductors. 311 Our results clearly reveal that this is attributed to standing 312 enokitake-like nanowire structures, vertically aligned config- 313 uration, and strong chemical bonding interactions between 314 standing nanowire films and elastomeric substrates. Together, 315 this leads to distinct elastic properties that have never been 316 observed for conventional bulk metal films or other nanoma- 317 terial networks (both vertically aligned and lying-down aligned 318 carbon-nanotube-based systems; see Table S2 in the 319 Supporting Information). We further demonstrate the 320 applications of our standing nanowire film in stretchable 321 supercapacitors and wearable e-skin sensors, beyond which we 322 
323 may find a myriad of additional applications in future 324 elastronics.

\section{METHODS}

326 Chemicals. Gold(III) chloride trihydrate $\left(\mathrm{HAuCl}_{4} \cdot 3 \mathrm{H}_{2} \mathrm{O}, 99.9 \%\right)$, 327 triisopropylsilane (99\%), 4-mercaptobenzoic acid (MBA, 90\%), 328 APTMS, sodium citrate tribasic dihydrate (99.0\%), L-ascorbic acid, 329 poly(vinyl alcohol) (PVA) powder, $\mathrm{H}_{3} \mathrm{PO}_{4}$, and ethanol (analytical 330 grade) were purchased from Sigma-Aldrich. All solutions were 331 prepared using deionized water (resistivity $>18 \mathrm{M} \Omega \cdot \mathrm{cm}^{-1}$ ). All 332 chemicals were used as received unless otherwise indicated. 333 Conductive wires were purchased from Adafruit.

334 Elastomeric Substrates. PDMS substrates were made by mixing 335 Sylgard 184 silicone elastomer base and curing agent at a weight ratio 336 of 10:1. The mixture was poured on a 6 in. flat-plate Petri dish using $3370.5 \mathrm{~mm}$ height shims as spacers and cured at $65^{\circ} \mathrm{C}$ for $2 \mathrm{~h}$ in an oven. 338 Ecoflex substrates were made by pouring Ecoflex curable silicone fluid 339 (Smooth-On Ecoflex 00-30) into a 6 in. flat-plate Petri dish and 340 curing under room temperature for $4 \mathrm{~h}$.

341 Synthesis of Standing Gold Nanowire Films. A modified seed342 mediated approach was used, as described in the literature. ${ }^{8}$ First, 2 $343 \mathrm{~nm}$ seed gold nanoparticles were synthesized. Briefly, $0.147 \mathrm{~mL}$ of 34 $344 \mathrm{mM}$ sodium citrate was added into a conical flask with $20 \mathrm{~mL}$ of $\mathrm{H}_{2} \mathrm{O}$ 345 under vigorous stirring. After $1 \mathrm{~min}, 600 \mu \mathrm{L}$ of ice-cold, freshly 346 prepared $0.1 \mathrm{M} \mathrm{NaBH}_{4}$ solution was added with stirring. The solution 347 turned brown immediately. The solution was then stirred for $5 \mathrm{~min}$ 348 and stored at $4{ }^{\circ} \mathrm{C}$ until needed.

349 To grow standing nanowires on substrates (e.g., Si wafer, Ecoflex), $350 \mathrm{O}_{2}$ plasma was applied to render the surfaces hydrophilic. Depending 351 on the types of substrates, the plasma treatment time varied from 2 to $35217 \mathrm{~min}$. Then the substrates were functionalized with an amino group 353 by silanization reaction with $5 \mathrm{mM}$ APTMS solution for $1 \mathrm{~h}$. APTMS354 modified substrates were immersed into excess citrate-stabilized Au 355 seed $(3-5 \mathrm{~nm})$ solution for $2 \mathrm{~h}$ to ensure the saturated adsorption of 356 gold seeds, followed by rinsing with water four times to remove the 357 weakly bound seed particles. Finally, seed-particle-anchored substrates 358 were in contact with a growth solution containing $980 \mu \mathrm{M} \mathrm{MBA}, 12$ $359 \mathrm{mM} \mathrm{HAuCl}$, and $29 \mathrm{mM} \mathrm{L}$-ascorbic acid, leading to the formation of 360 standing nanowire films. The length of nanowires depended on the 361 growth reaction time. Typical nanowire heights of $\sim 1.5, \sim 3.5, \sim 5, \sim 7$, 362 and $\sim 14 \mu \mathrm{m}$ were obtained by adjusting the growth time to $2,4,5,8$, 363 and 15 min, respectively.

364 Lying-Down Gold Nanowire Films. $\mathrm{HAuCl}_{4} \cdot 3 \mathrm{H}_{2} \mathrm{O}(44 \mathrm{mg})$ was 365 added into $40 \mathrm{~mL}$ of hexane, followed by addition of $1.5 \mathrm{~mL}$ of 366 oleylamine. After the gold salts were completely dissolved, $2.1 \mathrm{~mL}$ of 367 triisopropylsilane was added into the above solution. The resulting 368 solution was left to stand for 2 days without stirring at room 369 temperature until the color turned from yellow to dark, indicating the 370 formation of gold nanowires. The chemical residues were removed by 371 repeated centrifugation and thorough washing using ethanol/hexane $372(3 / 1, v / v)$ and finally concentrated to a $2 \mathrm{~mL}$ stock solution in 373 hexane. The lying-down gold nanowire films could then be obtained 374 by a simple drop-casting approach.

375 Vacuum-Evaporated Gold Film. A $100 \mathrm{~nm}$ gold film could be 376 obtained using an e-beam evaporator (Intlvac Nanochrome II, 10 $377 \mathrm{kV})$.

378 Characterization. SEM imaging was carried out using a FEI 379 Helios Nanolab 600 FIB-SEM operating at a voltage of $5 \mathrm{kV}$. The 380 sheet resistances of the standing enokitake-like nanowire-based gold 381 films were carried out on a Jandel four-point conductivity probe by 382 using a linear arrayed four-point head. To test the electromechanical 383 responses for strain and bending sensing, the two ends of the samples 384 were attached to motorized moving stages (THORLABS model 385 LTS150/M). Uniform stretching/bending cycles were applied by a 386 computer-based user interface (Thorlabs APT user), and the current 387 changes were measured by the Parstat 2273 electrochemical system 388 (Princeton Applied Research). For the analysis of detailed point load 389 or pressure responses, a computer-based user interface and a force 390 sensor (ATI Nano17 force/torque sensor) and a Maxon Brushless
DC motor using a high-resolution quadrature encoder $(15 \mu \mathrm{m}$ of 391 linear resolution) were used to apply an external point load or 392 pressure. Ecoflex with a thickness of $500 \mu \mathrm{m}$ was chosen as the 393 substrate of the standing nanowire film in a strain test. PET with a 394 thickness of $125 \mu \mathrm{m}$ was chosen as the substrate of the standing 395 nanowire film in a strain test. PDMS with a thickness of $1 \mathrm{~mm}$ was 396 chosen as the substrate of the standing nanowire film in a point load/ 397 pressure test. The reflectance $(R)$ data were collected from a 398 PerkinElmer UV-vis-NIR spectrophotometer (Lambda 1050) with 399 an integrating sphere setup.

400

Simulation. The finite element analysis model was implemented 401 in the ABAQUS 6.14/Standard software. Ecoflex substrate was 402 meshed using structured hex elements, whereas gold nanowires were 403 used a tetrahedral elements. There were a total of 2640 linear 404 hexahedral elements in the Ecoflex substrate and 106200 quadratic 405 tetrahedral elements in the gold nanowire section. The aspect ratio of 406 the gold nanowire was modeled at 100, with a length of $800 \mathrm{~nm}$ and a 407 diameter of $8 \mathrm{~nm}$. The elastic modulus and Poisson's ratio are 400408 $\mathrm{kPa}$ and 0.49 for the Ecoflex substrate and $70 \mathrm{GPa}$ and 0.42 for 409 nanowire, respectively. The boundary conditions were set by fixing 410 the left end of Ecoflex substrate and stretching uniaxially to $800 \% 411$ elongation. The contact condition between the nanowire layer and 412 Ecoflex substrate was assumed to be pinned using a tie constraint. 413

Elastic Supercapacitors. The standing enokitake-like nanowire- 414 based gold film was cut into small pieces with suitable shapes and 415 sizes. A gel solution that contained PVA powder (1.0 g) and $\mathrm{H}_{3} \mathrm{PO}_{4} 416$ $(1.0 \mathrm{~g})$ in water $(10.0 \mathrm{~mL})$ was coated on top of the prepared films 417 and dried in air for $5 \mathrm{~h}$. Then two such-prepared standing enokitake- 418 like nanowire-based gold film electrodes were assembled with 419 sandwiched electrolytes to form a symmetrical electrochemical 420 capacitor.

Wireless Facial Expression Monitoring. The circuit was 422 composed of nine standing enokitake-like nanowire-based gold film 423 sensors for measuring 11 facial muscle groups, and the supporting 424 circuit was constructed with $3.3 \mathrm{~V}$ power supply and $13330 \Omega 425$ resistors. After the standing enokitake-like nanowire-based gold film 426 sensors were mounted on the particularly targeted muscle groups on 427 the subject's face, electrical responses of each sensor were recorded. A 428 Bluetooth low energy technology was used to transfer the analogue 429 reading data of each sensor to an Android OS-equipped mobile device 430 (e.g., phone or pad style device). A specially designed app, already 431 installed on the mobile device, first went through a machine learning 432 session, which was referenced to the FACS library from Ekman's 433 group. The FACS contributes as the reference blueprint for pattern 434 recognitions to detect various facial expressions. This system was able 435 to process electrical responses from facial muscle groups in real time, 436 provided the baseline for measuring subject's detailed facial 437 movement, and eventually translated it to different emotional 438 expressions. The system was also able to create a data dictionary to 439 store the data based on the nine sensor readings to specific muscle 440 groups.

\section{ASSOCIATED CONTENT}

\section{S Supporting Information}

The Supporting Information is available free of charge on the 444 ACS Publications website at DOI: 10.1021/acsnano.8b05019. 445

Movie S1: Thin standing enokitake-like nanowire films 446 on the back of a human hand, stretching and releasing 447 (AVI)

Movie S2: Repeatable adhesion tape test (AVI) 449 Movie S3: Finite element analysis modeling of strain 450 deformation for standing nanowire film (AVI) 451 Movie S4: Wireless facial expression monitoring from 452 standing nanowire-based smart sensors (AVI) 453 Figures S1-S15, Notes S1 and S2, Tables S1 and S2, 454 and additional references (PDF) 455 


\section{AUTHOR INFORMATION}

\section{Corresponding Author}

458 *E-mail: wenlong.cheng@monash.edu.

\section{ORCID}

460 Lim Wei Yap: 0000-0003-3072-6307

461 Wenlong Cheng: 0000-0002-2346-4970

\section{Author Contributions}

${ }_{463}{ }^{\text {II }}$ Y.W. and S.G. contributed equally to this work.

\section{Notes}

465 The authors declare no competing financial interest.

\section{ACKNOWLEDGMENTS}

467 This work was performed in part at the Melbourne Centre for 468 Nanofabrication (MCN) in the Victorian Node of the 469 Australian National Fabrication Facility (ANFF). This work 470 is financially supported by ARC Discovery Projects 471 DP180101715 and LP160100521.

\section{REFERENCES}

473 (1) Rogers, J. A.; Someya, T.; Huang, Y. Materials and Mechanics 474 for Stretchable Electronics. Science 2010, 327, 1603-1607.

475 (2) Gong, S.; Cheng, W. Toward Soft Skin-Like Wearable and 476 Implantable Energy Devices. Adv. Energy Mater. 2017, 7, 1700648.

477 (3) Kim, D. H.; Xiao, J.; Song, J.; Huang, Y.; Rogers, J. A. 478 Stretchable, Curvilinear Electronics Based on Inorganic Materials. 479 Adv. Mater. 2010, 22, 2108-2124.

480 (4) Khang, D.-Y.; Jiang, H.; Huang, Y.; Rogers, J. A. A Stretchable 481 Form of Single-Crystal Silicon for High-Performance Electronics on 482 Rubber Substrates. Science 2006, 311, 208-212.

483 (5) Kaltenbrunner, M.; Sekitani, T.; Reeder, J.; Yokota, T.; Kuribara, 484 K.; Tokuhara, T.; Drack, M.; Schwödiauer, R.; Graz, I.; Bauer485 Gogonea, S.; et al. An Ultra-Lightweight Design for Imperceptible 486 Plastic Electronics. Nature 2013, 499, 458-463.

487 (6) Liu, Z.; Fang, S.; Moura, F.; Ding, J.; Jiang, N.; Di, J.; Zhang, M.; 488 Lepró, X.; Galvão, D.; Haines, C.; et al. Hierarchically Buckled 489 Sheath-Core Fibers for Superelastic Electronics, Sensors, and Muscles. 490 Science 2015, 349, 400-404.

491 (7) Xu, S.; Zhang, Y.; Cho, J.; Lee, J.; Huang, X.; Jia, L.; Fan, J. A.; 492 Su, Y.; Su, J.; Zhang, H.; et al. Stretchable Batteries with Self-Similar 493 Serpentine Interconnects and Integrated Wireless Recharging 494 Systems. Nat. Commun. 2013, 4, 1543.

495 (8) Song, Z.; Ma, T.; Tang, R.; Cheng, Q.; Wang, X.; Krishnaraju, 496 D.; Panat, R.; Chan, C. K.; Yu, H.; Jiang, H. Origami Lithium-Ion 497 Batteries. Nat. Commun. 2014, 5, 3140.

498 (9) White, M. S.; Kaltenbrunner, M.; Głowacki, E. D.; Gutnichenko, 499 K.; Kettlgruber, G.; Graz, I.; Aazou, S.; Ulbricht, C.; Egbe, D. A. M.; 500 Miron, M. C.; Major, Z.; Scharber, M. C.; Sekitani, T.; Someya, T.; 501 Bauer, S.; Sariciftci, N. S. Ultrathin, Highly Flexible and Stretchable 502 PLEDs. Nat. Photonics 2013, 7, 811-816.

503 (10) Niu, Z.; Dong, H.; Zhu, B.; Li, J.; Hng, H. H.; Zhou, W.; Chen, 504 X.; Xie, S. Highly Stretchable, Integrated Supercapacitors Based on 505 Single-Walled Carbon Nanotube Films with Continuous Reticulate 506 Architecture. Adv. Mater. 2013, 25, 1058-1064.

507 (11) Kim, K. S.; Zhao, Y.; Jang, H.; Lee, S. Y.; Kim, J. M.; Kim, K. S.; 508 Ahn, J.-H.; Kim, P.; Choi, J.-Y.; Hong, B. H. Large-Scale Pattern 509 Growth of Graphene Films for Stretchable Transparent Electrodes. 510 Nature 2009, 457, 706-710.

511 (12) Lipomi, D. J.; Vosgueritchian, M.; Tee, B. C.; Hellstrom, S. L.; 512 Lee, J. A.; Fox, C. H.; Bao, Z. Skin-Like Pressure and Strain Sensors 513 Based on Transparent Elastic Films of Carbon Nanotubes. Nat. 514 Nanotechnol. 2011, 6, 788-792.

515 (13) Wang, Y.; Zhu, C.; Pfattner, R.; Yan, H.; Jin, L.; Chen, S.; 516 Molina-Lopez, F.; Lissel, F.; Liu, J.; Rabiah, N. I.; et al. A Highly 517 Stretchable, Transparent, and Conductive Polymer. Sci. Adv. 2017, 3, 518 e 1602076.
(14) Liang, J.; Li, L.; Niu, X.; Yu, Z.; Pei, Q. Elastomeric Polymer 519 Light-Emitting Devices and Displays. Nat. Photonics 2013, 7, 817- 520 824.

(15) Sekitani, T.; Nakajima, H.; Maeda, H.; Fukushima, T.; Aida, T.; 522 Hata, K.; Someya, T. Stretchable Active-Matrix Organic Light- 523 Emitting Diode Display Using Printable Elastic Conductors. Nat. 524 Mater. 2009, 8, 494-499.

(16) Miyamoto, A.; Lee, S.; Cooray, N. F.; Lee, S.; Mori, M.; 526 Matsuhisa, N.; Jin, H.; Yoda, L.; Yokota, T.; Itoh, A.; Sekino, M.; 527 Kawasaki, H.; Ebihara, T.; Amagai, M.; Someya, T. Inflammation- 528 Free, Gas-Permeable, Lightweight, Stretchable on-Skin Electronics 529 with Nanomeshes. Nat. Nanotechnol. 2017, 12, 907-913.

530

(17) Matsuhisa, N.; Inoue, D.; Zalar, P.; Jin, H.; Matsuba, Y.; Itoh, 531 A.; Yokota, T.; Hashizume, D.; Someya, T. Printable Elastic 532 Conductors by in Situ Formation of Silver Nanoparticles from Silver 533 Flakes. Nat. Mater. 2017, 16, 834-840.

534

(18) Keplinger, C.; Sun, J.-Y.; Foo, C. C.; Rothemund, P.; 535 Whitesides, G. M.; Suo, Z. Stretchable, Transparent, Ionic 536 Conductors. Science 2013, 341, 984-987.

(19) Muth, J. T.; Vogt, D. M.; Truby, R. L.; Mengüç, Y.; Kolesky, D. 538 B.; Wood, R. J.; Lewis, J. A. Embedded 3d Printing of Strain Sensors 539 within Highly Stretchable Elastomers. Adv. Mater. 2014, 26, 6307- 540 6312.

(20) Tavakoli, M.; Malakooti, M. H.; Paisana, H.; Ohm, Y.; Green 542 Marques, D.; Alhais Lopes, P.; Piedade, A. P.; de Almeida, A. T.; 543 Majidi, C. Egain-Assisted Room-Temperature Sintering of Silver 544 Nanoparticles for Stretchable, Inkjet-Printed, Thin-Film Electronics. 545 Adv. Mater. 2018, 30, 1801852.

(21) Wang, J.; Cai, G.; Li, S.; Gao, D.; Xiong, J.; Lee, P. S. Printable 547 Superelastic Conductors with Extreme Stretchability and Robust 548 Cycling Endurance Enabled by Liquid-Metal Particles. Adv. Mater. 549 2018, 30, 1706157.

(22) Jin, J.; Lee, D.; Im, H. G.; Han, Y. C.; Jeong, E. G.; Rolandi, M.; 551 Choi, K. C.; Bae, B. S. Chitin Nanofiber Transparent Paper for 552 Flexible Green Electronics. Adv. Mater. 2016, 28, 5169-5175. 553

(23) Chen, G.; Matsuhisa, N.; Liu, Z.; Qi, D.; Cai, P.; Jiang, Y.; Wan, 554 C.; Cui, Y.; Leow, W. R.; Liu, Z.; et al. Plasticizing Silk Protein for on- 555 Skin Stretchable Electrodes. Adv. Mater. 2018, 30, 1800129.

(24) Wang, Y.; Gong, S.; Wang, S. J.; Simon, G. P.; Cheng, W. 557 Volume-Invariant Ionic Liquid Microbands as Highly Durable 558 Wearable Biomedical Sensors. Mater. Horiz. 2016, 3, 208-213.

(25) Xu, F.; Zhu, Y. Highly Conductive and Stretchable Silver 560 Nanowire Conductors. Adv. Mater. 2012, 24, 5117-5122.

(26) Gong, S.; Lai, D. T. H.; Su, B.; Si, K. J.; Ma, Z.; Yap, L. W.; 562 Guo, P.; Cheng, W. Highly Stretchy Black Gold E-Skin Nanopatches 563 as Highly Sensitive Wearable Biomedical Sensors. Adv. Electron. 564 Mater. 2015, 1, 1400063.

(27) Lee, P.; Lee, J.; Lee, H.; Yeo, J.; Hong, S.; Nam, K. H.; Lee, D.; 566 Lee, S. S.; Ko, S. H. Highly Stretchable and Highly Conductive Metal 567 Electrode by Very Long Metal Nanowire Percolation Network. Adv. 568 Mater. 2012, 24, 3326-3332.

(28) Song, J.; Li, J.; Xu, J.; Zeng, H. Superstable Transparent 570 Conductive $\mathrm{Cu} @ \mathrm{Cu}_{4} \mathrm{Ni}$ Nanowire Elastomer Composites against 571 Oxidation, Bending, Stretching, and Twisting for Flexible and 572 Stretchable Optoelectronics. Nano Lett. 2014, 14, 6298-6305. 573

(29) Lee, P.; Ham, J.; Lee, J.; Hong, S.; Han, S.; Suh, Y. D.; Lee, S. 574 E.; Yeo, J.; Lee, S. S.; Lee, D.; Ko, S. H. Highly Stretchable or 575 Transparent Conductor Fabrication by a Hierarchical Multiscale 576 Hybrid Nanocomposite. Adv. Funct. Mater. 2014, 24, 5671-5678. 577

(30) Yamada, T.; Hayamizu, Y.; Yamamoto, Y.; Yomogida, Y.; Izadi- 578 Najafabadi, A.; Futaba, D. N.; Hata, K. A Stretchable Carbon 579 Nanotube Strain Sensor for Human-Motion Detection. Nat. Nano- 580 technol. 2011, 6, 296-301.

(31) Lee, P.; Lee, J.; Lee, H.; Yeo, J.; Hong, S.; Nam, K. H.; Lee, D.; 582 Lee, S. S.; Ko, S. H. Highly Stretchable and Highly Conductive Metal 583 Electrode by Very Long Metal Nanowire Percolation Network. Adv. 584 Mater. 2012, 24, 3326-3332.

(32) Han, S.; Hong, S.; Ham, J.; Yeo, J.; Lee, J.; Kang, B.; Lee, P.; 586 Kwon, J.; Lee, S. S.; Yang, M. Y.; et al. Fast Plasmonic Laser 587 
588 Nanowelding for a Cu-Nanowire Percolation Network for Flexible 589 Transparent Conductors and Stretchable Electronics. Adv. Mater. 590 2014, 26, 5808-5814.

591 (33) Yamada, T.; Hayamizu, Y.; Yamamoto, Y.; Yomogida, Y.; Izadi592 Najafabadi, A.; Futaba, D. N.; Hata, K. A Stretchable Carbon 593 Nanotube Strain Sensor for Human-Motion Detection. Nat. Nano594 technol. 2011, 6, 296-301.

595 (34) Sun, H.; You, X.; Deng, J.; Chen, X.; Yang, Z.; Chen, P.; Fang, 596 X.; Peng, H. A Twisted Wire-Shaped Dual-Function Energy Device 597 for Photoelectric Conversion and Electrochemical Storage. Angew. 598 Chem., Int. Ed. 2014, 53, 6664-6668.

599 (35) Kim, K. K.; Hong, S.; Cho, H. M.; Lee, J.; Suh, Y. D.; Ham, J.; $600 \mathrm{Ko}, \mathrm{S}$. H. Highly Sensitive and Stretchable Multidimensional Strain 601 Sensor with Prestrained Anisotropic Metal Nanowire Percolation 602 Networks. Nano Lett. 2015, 15, 5240-5247.

603 (36) Jeong, C. K.; Lee, J.; Han, S.; Ryu, J.; Hwang, G. T.; Park, D. Y.; 604 Park, J. H.; Lee, S. S.; Byun, M.; Ko, S. H.; et al. A Hyper-Stretchable 605 Elastic-Composite Energy Harvester. Adv. Mater. 2015, 27, 28666062875

607 (37) Moon, H.; Lee, H.; Kwon, J.; Suh, Y. D.; Kim, D. K.; Ha, I.; 608 Yeo, J.; Hong, S.; Ko, S. H. Ag/Au/Polypyrrole Core-Shell Nanowire 609 Network for Transparent, Stretchable and Flexible Supercapacitor in 610 Wearable Energy Devices. Sci. Rep. 2017, 7, 41981.

611 (38) Lee, J.; An, K.; Won, P.; Ka, Y.; Hwang, H.; Moon, H.; Kwon, 612 Y.; Hong, S.; Kim, C.; Lee, C.; et al. A Dual-Scale Metal Nanowire 613 Network Transparent Conductor for Highly Efficient and Flexible 614 Organic Light Emitting Diodes. Nanoscale 2017, 9, 1978-1985.

615 (39) Park, J. H.; Han, S.; Kim, D.; You, B. K.; Joe, D. J.; Hong, S.; 616 Seo, J.; Kwon, J.; Jeong, C. K.; Park, H. J.; et al. Plasmonic-Tuned 617 Flash $\mathrm{Cu}$ Nanowelding with Ultrafast Photochemical-Reducing and 618 Interlocking on Flexible Plastics. Adv. Funct. Mater. 2017, 27, 6191701138 .

620 (40) Jeong, S.; Cho, H.; Han, S.; Won, P.; Lee, H.; Hong, S.; Yeo, J.; 621 Kwon, J.; Ko, S. H. High Efficiency, Transparent, Reusable, and 622 Active PM2.5 Filters by Hierarchical Ag Nanowire Percolation 623 Network. Nano Lett. 2017, 17, 4339-4346.

624 (41) Han, S.; Hong, S.; Yeo, J.; Kim, D.; Kang, B.; Yang, M. Y.; Ko, 625 S. H. Nanorecycling: Monolithic Integration of Copper and Copper 626 Oxide Nanowire Network Electrode through Selective Reversible 627 Photothermochemical Reduction. Adv. Mater. 2015, 27, 6397-6403. 628 (42) Hong, S.; Lee, H.; Lee, J.; Kwon, J.; Han, S.; Suh, Y. D.; Cho, 629 H.; Shin, J.; Yeo, J.; Ko, S. H. Highly Stretchable and Transparent 630 Metal Nanowire Heater for Wearable Electronics Applications. Adv. 631 Mater. 2015, 27, 4744-4751.

632 (43) Jung, J.; Lee, H.; Ha, I.; Cho, H.; Kim, K. K.; Kwon, J.; Won, 633 P.; Hong, S.; Ko, S. H. Highly Stretchable and Transparent 634 Electromagnetic Interference Shielding Film Based on Silver Nano635 wire Percolation Network for Wearable Electronics Applications. ACS 636 Appl. Mater. Interfaces 2017, 9, 44609-44616.

637 (44) Gong, S.; Zhao, Y.; Yap, L. W.; Shi, Q.; Wang, Y.; Bay, J. A. P.; 638 Lai, D. T.; Uddin, H.; Cheng, W. Fabrication of Highly Transparent 639 and Flexible Nanomesh Electrode via Self-Assembly of Ultrathin Gold 640 Nanowires. Adv. Electron. Mater. 2016, 2, 1600121.

641 (45) Ho, M. D.; Ling, Y.; Yap, L. W.; Wang, Y.; Dong, D.; Zhao, Y.; 642 Cheng, W. Percolating Network of Ultrathin Gold Nanowires and 643 Silver Nanowires toward "Invisible" Wearable Sensors for Detecting 644 Emotional Expression and Apexcardiogram. Adv. Funct. Mater. 2017, $64527,1700845$.

646 (46) Ho, M. D.; Liu, Y.; Dong, D.; Zhao, Y.; Cheng, W. Fractal Gold 647 Nanoframework for Highly Stretchable Transparent Strain-Insensitive 648 Conductors. Nano Lett. 2018, 18, 3593.

649 (47) Lu, N.; Wang, X.; Suo, Z.; Vlassak, J. Metal Films on Polymer 650 Substrates Stretched Beyond 50\%. Appl. Phys. Lett. 2007, 91, 221909. 651 (48) He, J.; Wang, Y.; Feng, Y.; Qi, X.; Zeng, Z.; Liu, Q.; Teo, W. S.; 652 Gan, C. L.; Zhang, H.; Chen, H. Forest of Gold Nanowires: A New 653 Type of Nanocrystal Growth. ACS Nano 2013, 7, 2733-2740.

654 (49) Amjadi, M.; Pichitpajongkit, A.; Lee, S.; Ryu, S.; Park, I. Highly 655 Stretchable and Sensitive Strain Sensor Based on Silver Nanowire656 Elastomer Nanocomposite. ACS Nano 2014, 8, 5154-5163.
(50) Kim, Y.; Zhu, J.; Yeom, B.; Di Prima, M.; Su, X.; Kim, J.-G.; 657 Yoo, S. J.; Uher, C.; Kotov, N. A. Stretchable Nanoparticle 658 Conductors with Self-Organized Conductive Pathways. Nature 659 2013, 500, 59-63.

(51) Chun, K. Y.; Oh, Y.; Rho, J.; Ahn, J. H.; Kim, Y. J.; Choi, H. R.; 661 Baik, S. Highly Conductive, Printable and Stretchable Composite 662 Films of Carbon Nanotubes and Silver. Nat. Nanotechnol. 2010, 5, 663 853-857.

(52) Moon, G. D.; Lim, G. H.; Song, J. H.; Shin, M.; Yu, T.; Lim, B.; 665 Jeong, U. Highly Stretchable Patterned Gold Electrodes Made of Au 666 Nanosheets. Adv. Mater. 2013, 25, 2707-2712.

667

(53) Matsuhisa, N.; Kaltenbrunner, M.; Yokota, T.; Jinno, H.; 668 Kuribara, K.; Sekitani, T.; Someya, T. Printable Elastic Conductors 669 with a High Conductivity for Electronic Textile Applications. Nat. 670 Commun. 2015, 6, 7461 .

671

(54) Tybrandt, K.; Voros, J. Fast and Efficient Fabrication of 672 Intrinsically Stretchable Multilayer Circuit Boards by Wax Pattern 673 Assisted Filtration. Small 2016, 12, 180-184.

674

(55) Park, M.; Im, J.; Shin, M.; Min, Y.; Park, J.; Cho, H.; Park, S.; 675 Shim, M. B.; Jeon, S.; Chung, D. Y.; Bae, J.; Park, J.; Jeong, U.; Kim, 676 K. Highly Stretchable Electric Circuits from a Composite Material of 677 Silver Nanoparticles and Elastomeric Fibres. Nat. Nanotechnol. 2012, 678 7, 803-809.

(56) Hu, L.; Yuan, W.; Brochu, P.; Gruner, G.; Pei, Q. Highly 680 Stretchable, Conductive, and Transparent Nanotube Thin Films. 681 Appl. Phys. Lett. 2009, 94, 161108.

(57) Mates, J. E.; Bayer, I. S.; Palumbo, J. M.; Carroll, P. J.; 683 Megaridis, C. M. Extremely Stretchable and Conductive Water- 684 Repellent Coatings for Low-Cost Ultra-Flexible Electronics. Nat. 685 Commun. 2015, 6, 8874

(58) Guo, R.; Yu, Y.; Xie, Z.; Liu, X.; Zhou, X.; Gao, Y.; Liu, Z.; 687 Zhou, F.; Yang, Y.; Zheng, Z. Matrix-Assisted Catalytic Printing for 688 the Fabrication of Multiscale, Flexible, Foldable, and Stretchable 689 Metal Conductors. Adv. Mater. 2013, 25, 3343-3350.

690

(59) Guo, R.; Yu, Y.; Zeng, J.; Liu, X.; Zhou, X.; Niu, L.; Gao, T.; Li, 691 K.; Yang, Y.; Zhou, F.; et al. Biomimicking Topographic Elastomeric 692 Petals (E-Petals) for Omnidirectional Stretchable and Printable 693 Electronics. Adv. Sci. 2015, 2, 1400021.

(60) Wang, X.; Hu, H.; Shen, Y.; Zhou, X.; Zheng, Z. Stretchable 695 Conductors with Ultrahigh Tensile Strain and Stable Metallic 696 Conductance Enabled by Prestrained Polyelectrolyte Nanoplatforms. 697 Adv. Mater. 2011, 23, 3090-3094.

(61) Yu, Y.; Yan, C.; Zheng, Z. Polymer-Assisted Metal Deposition 699 (PAMD): A Full-Solution Strategy for Flexible, Stretchable, 700 Compressible, and Wearable Metal Conductors. Adv. Mater. 2014, 701 $26,5508-5516$.

(62) Ekman, P.; Friesen, W. V. Unmasking the Face: A Guide to 703 Recognizing Emotions from Facial Clues; ISHK: Los Altos, CA, 2003. 704 